\title{
Alternative Strategies in Thalassemia: Focus on Thalidomide
}

\author{
R. Naithani ${ }^{1} \cdot$ P. Jeyaraman ${ }^{1} \cdot$ M. Mahapatra ${ }^{1}$
}

Published online: 5 May 2020

(C) Indian Society of Hematology and Blood Transfusion 2020

India has one of the largest burdens of children living with transfusion dependent thalassemia with prevalence of beta thalassemia carriers in India is 3-17\% [1]. Bone marrow transplantation is the only curative option. Matched sibling donor availability and high cost of transplant usually drive it out of reach for majority of patients. Gene therapy and gene editing although offer a chance of cure is still not available in India. Regular transfusion and iron chelation remain a reality for large majority of Indian patients. Safe and adequate transfusion of blood, high cost and poor compliance with chelation therapy pose a challenge for optimal management of these patients. Alternative treatment options that focus on correction of globin chain imbalance, ineffective erythropoiesis and improving how the body handles iron are increasingly being studied [2]. Increase in $\gamma$-globin reduces $\alpha$ chain imbalance and ineffective erythropoiesis. A number of traditional agents have been identified to up regulate $\mathrm{Hb} F$ including sodium butyrate, azacytidine, decitabine and hydroxyurea. Newer small molecule pharmacological agents against specific molecular targets (HDAC, DNMT1A, KDM1A, PRMT5, EHMT2 and ISWI) for $\gamma$-globin induction are in the pipeline [3]. However, these $\gamma$-globin inducers have shown only a modest effect in the majority of $\beta$-thalassaemia patients and are associated with some degree of toxicity. These agents except hydroxyurea are not being used routinely in clinical practice. Transforming growth factor $\beta$ superfamily of ligands have been shown to inhibit late stage haematopoiesis. Luspatercept, novel recombinant fusion protein acts as a trap to these ligands and has been

\footnotetext{
M. Mahapatra

mrmahapatra@hotmail.com

1 New Delhi, India
}

shown to be effective in patients with $\beta$-thalassemia. Other agents in development include sotatercept, macrophage inhibitiors, JAK2 inhibitor-ruxolitinib. Thalidomide is known for its immune-modulating and anti-angiogenic properties [2]. In recent years, thalidomide has been shown to increase hemoglobin $F$ levels through inducing $\gamma$-globin mRNA expression in a dose-dependent manner by increasing intracellular reactive oxygen species [4]. Few small case series and retrospective studies have shown remarkable response to thalidomide therapy in patients with thalassemia major and intermedia [5, 6]. In this issue, $\mathrm{Nag}$ et al. [7], retrospectively studied the efficacy of thalidomide in 21 transfusion dependent E-Beta thalassemia patients. Fifteen patients achieved complete response, 1 patient had partial response and 5 were nonresponders. The median time to response in these patients was 1 month. Yasin et al. demonstrated sustained responses in $75.7 \%$ of patients in a series of 37 patients with symptomatic $\beta$-thalassemia (including 14 transfusion dependent patients) [8]. The median time to response was 1.7 months. Significant increase in hemoglobin from 7.8 to $9.9 \mathrm{~g} / \mathrm{dL}(p<0.001)$ was documented in non-transfusion dependent patients while the yearly blood units transfused dropped from 27 to 7.8 in transfusion dependent patients $(p=0.001)$. Recently, high response rates have been demonstrated in 25 (15 thalassemia intermedia and 10 thalassemia major) Indian patients with combination therapy of hydroxyurea and thalidomide [9]. Twenty percent of patients in the study had undergone splenectomy. Transfusion independence was achieved in $78.6 \%$ of patients with thalassemia intermedia and $62.5 \%$ of thalassemia major patients. In a case series from China 7 patients of thalassemia intermedia, ( 3 on transfusion therapy) had received thalidomide $(50 \mathrm{mg})$. At 3 months, hemoglobin concentration increased significantly $(\geq 2 \mathrm{~g} / \mathrm{dl})$ in three 
non-transfused patients and moderately (1-2 g/dl) in one non-transfused patient [10]. In patients on transfusion therapy, transfusion was terminated in one patient and frequency of transfusion decreased $>50 \%$ in the other two patients, accompanied by an increase in the average hemoglobin concentration. Masera et al. [5], reported a patient with repeated massive post transfusion acute hemolysis due to alloimmunisation, who dramatically responded, becoming transfusion independent after thalidomide therapy with hydroxyurea. In all these studies, thalidomide has been well tolerated with minimal adverse events. Thalidomide does offer a hope of convenient, effective and affordable therapy in thalassemia patients who are unable to undergo allogeneic stem cell transplant or as a bridge to transplant reducing transfusion requirements thereby reducing iron overload. However, so far data is available in small case series only. Caution should be exercised in young female patients of reproductive age group to avoid potential teratogenicity. Could thalidomide be an affordable alternative to transfusion chelation regime or at least partly offset it? Large prospective randomized controlled studies are required to answer this very question.

\section{Compliance with Ethical Standards}

Conflict of interest Authors declare that they have no conflict of interest.

Informed Consent Patient gave informed consent for his treatment. This study does not involve any research on humans.

\section{References}

1. Ray GK, Jena RK (2019) Spectrum of hemoglobinopathies: a new revelation in a tertiary care hospital of Odisha. Indian $\mathbf{J}$ Hematol Blood Transfus 35(3):513-517
2. Cappellini MD, Porter JB, Viprakasit V, Taher AT (2018) A paradigm shift on beta-thalassaemia treatment: how will we manage this old disease with new therapies? Blood Rev 32(4):300-311

3. Lavelle D, Engel JD, Saunthararajah Y (2018) Fetal hemoglobin induction by epigenetic drugs. Semin Hematol 55(2):60-67

4. Aerbajinai W, Zhu J, Gao Z, Chin K, Rodgers GP (2007) Thalidomide induces gamma-globin gene expression through increased reactive oxygen species-mediated p38 MAPK signaling and histone $\mathrm{H} 4$ acetylation in adult erythropoiesis. Blood 110(8):2864-2871

5. Masera N, Tavecchia L, Capra M, Cazzaniga G, Vimercati C, Pozzi L et al (2010) Optimal response to thalidomide in a patient with thalassaemia major resistant to conventional therapy. Blood Transfus 8(1):63-65

6. Fozza C, Pardini S, Giannico DB, Targhetta C, Di Tucci AA, Dessalvi P et al (2015) Dramatic erythroid response to low-dose thalidomide in two patients with transfusion independent thalassemia and severe post-transfusional alloimmune hemolysis. Am J Hematol 90(7):E141

7. Nag A, Radhakrishnan VS, Kumar J et al (2020) Thalidomide in patients with transfusion-dependent E-beta thalassemia refractory to hydroxyurea: a single-center experience. Indian J Hematol Blood Transfus. https://doi.org/10.1007/s12288-020-01263-2

8. Yassin AK (2019) Promising response to thalidomide in symptomatic $\beta$-thalassemia. Indian J Hematol Blood Transfus. https:// doi.org/10.1007/s12288-019-01231-5

9. Shah S, Sheth R, Shah K, Patel K (2019) Safety and effectiveness of thalidomide and hydroxyurea combination in beta-thalassaemia intermedia and major: a retrospective pilot study. $\mathrm{Br} \mathrm{J}$ Haematol. https://doi.org/10.1111/bjh.16272

10. Li Y, Ren Q, Zhou Y, Li P, Lin W, Yin X (2018) Thalidomide has a significant effect in patients with thalassemia intermedia. Hematology 23(1):50-54

Publisher's Note Springer Nature remains neutral with regard to jurisdictional claims in published maps and institutional affiliations. 